\title{
Real time monitoring method for the longitudinal settlement of shield tunnel using wireless inclinometer
}

\author{
YIN Jianguo ${ }^{1, a^{*}}$, HUANG Hongwei ${ }^{1, b}$ \\ ${ }^{1}$ Department of Geotechnical Engineering, Tongji University, Shanghai, P.R.China \\ ayjgkyo@163.com, bhuanghw@tongji.edu.cn
}

Keyword: longitudinal settlement; inclinometer; real time monitoring; wireless transmission; shield tunnel;

\begin{abstract}
At present, many common diseases including seepage, crack, segment staggering and longitudinal settlement, appear in the underground structure of urban track traffic. As one of main factors resulting in other diseases, longitudinal settlement can put great adverse influence on longitudinal performance of shield tunnel. So it is key to monitor longitudinal settlement accurately and timely. However, there are many shortcomings for the existed monitoring methods around the world. This paper presents a kind of real time monitoring method for longitudinal settlement of shield tunnel using Wireless Sensor Network (WSN) and Micro-Electro-Mechanic System (MEMS). Feasibility of the method is verified through MATLAB programming simulation, and the related technical parameters are determined based on present monitoring requirement.
\end{abstract}

\section{Introduction}

It is important to do a timely and accurate monitoring on the longitudinal settlement of shield tunnel, and there are many kinds of monitoring methods for the longitudinal settlement of shield tunnel structure, but these methods all own many defects which need to be improved. Besides, some emerging technologies, such as WSN technology and MEMS sensor, offer us new ways to do monitoring for underground engineering depending on their unique advantages.

It is relatively late for the application of WSN and MEMS in geotechnical engineering compared with other engineering fields. In 2007, Li and Liu brought the WSN into the coal mine monitoring to avoid its collapse. [1] In 2009, Yoshinori Iwasaki presented a introduction of sensor technologies in frontier domains including MEMS, geochemical sensor, TDR humility sensor and so on.[2] Recent years, how to keep tunnel structure in safe and healthy state has gradually become a heated issue with the rapid development of urban track traffic system, which attracts scholars' attention. In 2010, Frank Stajano et al. applied the WSN into the tunnel structure monitoring and presented the existing problems and potential solutions in the aspect of sensor's hardware, wireless transition, layout of nodes and so on, which has guidance function for later related research.[3] At the same year, Kenichi Soga gave a brief introduction about emerging technologies in tunnel structure monitoring including image recognition, MEMS techniques, optical fiber sensors, WSN techniques and so on, and did field test in London tunnel.[4] In 2012, Zhang Wei and Huang Hongwei did theoretical and experimental research on the measurement of transverse convergence deformation using wireless inclination sensor.[5] In 2013, F. WANG et al. did longitudinal deformation monitoring using FBG technique for a part of the Shanghai metro line 2 which was under influence by adjacent excavation, and comparison and validation was conducted by adopting hydrostatic levelling instruments. [6]

But there is still blank in the research on longitudinal settlement monitoring of shield tunnel based on WSN and MEMS around the world. This paper presents a kind of real time monitoring method for longitudinal settlement of shield tunnel using Wireless Sensor Network (WSN) and Micro-Electro-Mechanic System (MEMS). Feasibility of the method is verified through MATLAB programming simulation, and the related technical parameters are determined based on present monitoring requirement. 


\section{The existing monitoring methods}

For the longitudinal settlement monitoring of shield tunnel, there exist following methods:

(1) Hydrostatic levelling method

Hydrostatic levelling method can realize automation and digitalization in some degree, and is widely used for longitudinal settlement monitoring of shield tunnel. But its big volume, inconvenience in installation, unsuitable for big differential elevation section are main defects of this method. Besides it is also influenced by gravity and air pressure. [7-8]

(2) Manual levelling method

Manual levelling method is adopted extensively because of its maturity and low cost. But this method needs manual operation in the process of measurement resulting in low efficiency, so it cannot do 24-hour monitoring due to the train running.

(3) Electronic level meter

Electronic level meter has characteristics of high resolution and good stability, and it is convenient for installation. Besides, it can do real time monitoring even when tunnel is in normal operation. But because numerous instruments are needed and the cost of electronic level meter is not quite low, so total cost of this method is high and is its key defect. [9-10]

As the existing monitoring methods for longitudinal settlement of shield tunnel has abovementioned defects, this paper presents a kind of real time monitoring method for longitudinal settlement of shield tunnel using Wireless Sensor Network (WSN) and Micro-Electro-Mechanic System (MEMS).

\section{Description for the longitudinal settlement monitoring method using inclinometer}

As the longitudinal stiffness of shield tunnel structure is relatively small, so great many factors including curved jacking, rectification and soil disturbance in construction stage, long-term settlement and train vibration in operation stage, will result in longitudinal settlement of shield tunnel. Shield tunnel structure consists of precast reinforced concrete segments, and the longitudinal settlement of shield tunnel is not continuous because of the joints. This paper tentatively plans to fix PVC inclinometer tube on the lateral segment of shield tunnel along the longitudinal direction. And we suppose that inclinometer tube deforms in accordance with longitudinal deformation of shield tunnel. Longitudinal settlement of shield tunnel now can be converted into the longitudinal deformation of the inclinometer tube. An instrument powered by itself is designed to move inside the tube. The instrument owns MEMS inclinometer and radio communication device and can measure the angle between the axis of tube and the horizontal level during moving along the tube, as is shown in Fig. 1. Monitoring data was transmitted to workstation through radio communication device at the same time. And longitudinal settlement curve of shield tunnel can be obtained based on the data calculation, as shown in Fig. 2.

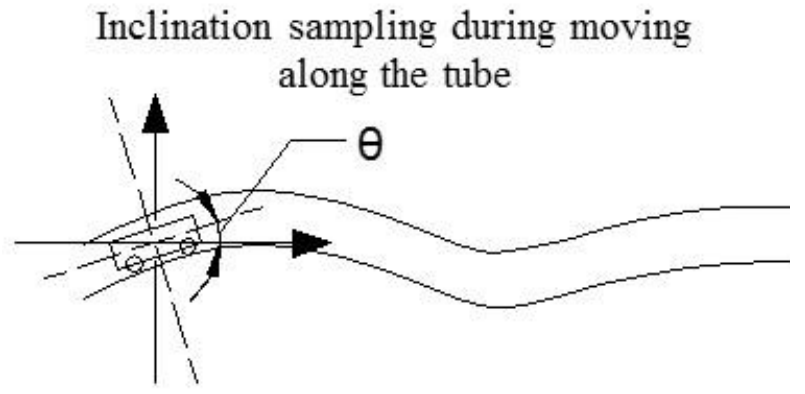

Fig. 1 Inclination sampling during moving along the tube
Real time monitoring method for longitudinal settlement of shield tunnel using wireless inclinometer

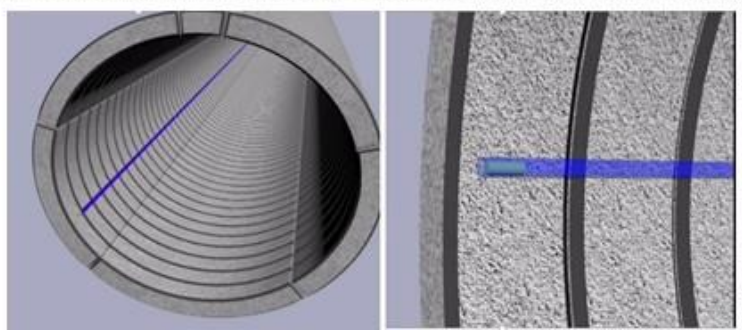

Fig. 2 Real time monitoring method for longitudinal settlement of shield tunnel using wireless inclinometer 


\section{Longitudinal settlement curve fitting based on inclinometer data}

Outline of Longitudinal Settlement Curve Calculation Based on Inclinometer Data. After getting the inclination data, we should convert it to longitudinal settlement curve of shield tunnel. The site monitoring data of some section of Shanghai metro line 1 is used to do research on reasonable curve fitting method. Cubic spline interpolation is used to get the actual settlement curve based on site measuring data. A series inclination data (Fig. 3) is also obtained using the measuring method abovementioned and is converted into longitudinal settlement curve (Fig. 4) using integral principle. The error is simulated by normal white noise.

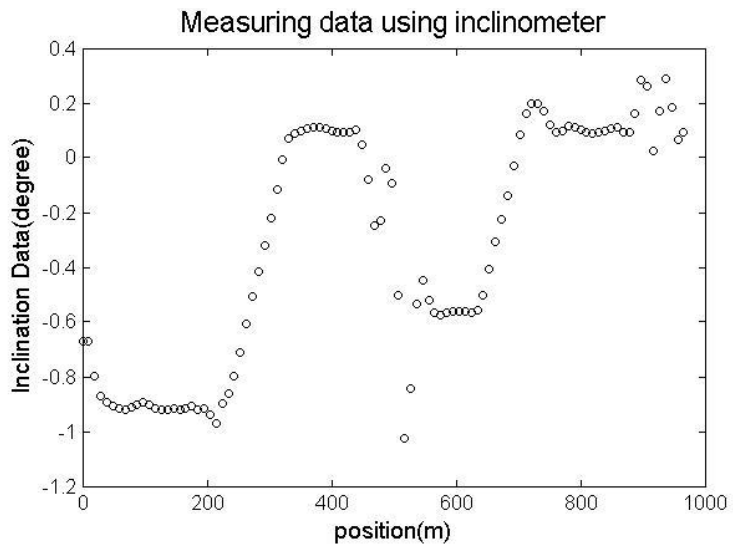

Fig. 3 Measuring data using inclinometer

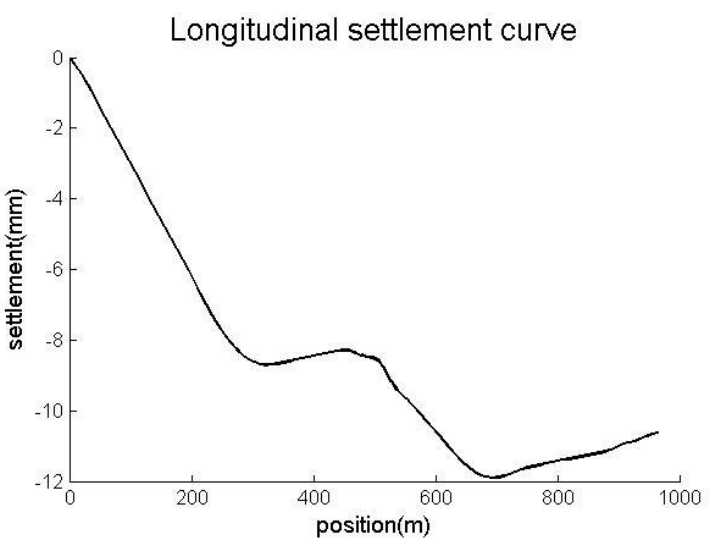

Fig. 4 Longitudinal settlement curve

Curve Fitting Method for Longitudinal Settlement Curve of Shield Tunnel Using Inclinometer. The data obtained by inclinometer is the inclination at the point of inclinometer and can be converted into the longitudinal settlement curve using integral principle.

The inclination data measured by inclinometer is $\left(x_{i}, y_{i}^{\prime}\right), i=0,1, \ldots, n-1, n$, and the longitudinal inclination curve is $y=f(x)=L^{\prime}(x)$, so the longitudinal settlement curve of shield tunnel is $y=L(x)=\int_{x_{0}}^{x} f(\xi) d \xi$

When the measuring points are equidistant nodes, the curve fitting equations are shown as follows (Eq. 1 Eq. 6).

Curve fitting method based on mid-point integration equation.

$$
\begin{aligned}
& L\left(x_{n}\right)=\frac{1}{2} y_{0}^{\prime} \Delta l+\frac{1}{2} y_{n}^{\prime} \Delta l+\sum_{k=1}^{n-1} y_{k}^{\prime} \Delta l+R_{n}\left(x_{n}\right) \\
& R_{n}\left(x_{n}\right)=\frac{1}{8} \Delta l^{2} f^{\prime}\left(\xi_{0}\right)+\frac{1}{8} \Delta l^{2} f^{(1)}\left(\xi_{n}\right)+\sum_{k=1}^{n-1} \frac{1}{24} \Delta l^{3} f^{(2)}\left(\xi_{k}\right), \xi_{k} \in\left[x_{k}, x_{k+1}\right]
\end{aligned}
$$

Curve fitting method based on trapezoid integration equation.

$$
\begin{aligned}
& L\left(x_{n}\right)=\sum_{k=0}^{n-1} \frac{1}{2}\left(y_{k}^{\prime}+y_{k+1}^{\prime}\right) \Delta l+R_{n}\left(x_{n}\right) \\
& R_{n}\left(x_{n}\right)=\sum_{k=0}^{n-1} \frac{1}{12} \Delta l^{3} f^{(2)}\left(\xi_{k}\right), \xi_{k} \in\left[x_{k}, x_{k+1}\right]
\end{aligned}
$$

Curve fitting method based on Simpson integration equation. 


$$
\begin{aligned}
& L\left(x_{n}\right)=\left\{\begin{array}{l}
\sum_{k=0}^{n-2} \frac{1}{3}\left(y_{k}^{\prime}+4 y_{k+1}^{\prime}+y_{k+2}^{\prime}\right) \Delta l+R_{n}\left(x_{n}\right) \quad \text { when } \mathrm{n} \text { is even number } \\
\sum_{k=1}^{n-2} \frac{1}{3}\left(y_{k}^{\prime}+4 y_{k+1}^{\prime}+y_{k+2}^{\prime}\right) \Delta l+\frac{1}{2}\left(y_{1}^{\prime}+y_{2}^{\prime}\right) \Delta l+R_{n}\left(x_{n}\right) \quad \text { when } \mathrm{n} \text { is odd number }
\end{array}\right. \\
& R_{n}\left(x_{n}\right)=\left\{\begin{array}{l}
\sum_{k=0}^{n-1}-\frac{1}{90} \Delta l^{5} f^{(4)}\left(\xi_{k}\right), \xi_{k} \in\left[x_{k}, x_{k+1}\right] \text { when } \mathrm{n} \text { is even number } \\
\sum_{k=1}^{n-1}-\frac{1}{90} \Delta l^{5} f^{(4)}\left(\xi_{k}\right)+\frac{1}{12} \Delta l^{3} f^{(2)}\left(\xi_{0}\right), \xi_{k} \in\left[x_{k}, x_{k+1}\right] \text { when } \mathrm{n} \text { is odd number }
\end{array}\right.
\end{aligned}
$$

Apart from the three methods, the curve fitting mehod based on cubic spline is also widely used.

Curve fitting error is influenced by many factors such as curve fitting method, inclinometer accuracy and sampling space, which will be discussed below.

Analysis of Various Curve Fitting Methods. Longitudinal settlement curve is obtained based on various curve fitting methods and the same series of measuring data. According to the comparison between the calculated settlement curve and the actual settlement curve, we can get the error of various curve fitting methods as shown in Fig. 5.

Fig. 5 indicates that the error of the curve fitting method based on the cubic spline method is the minimum among the four curve fitting methods. With the inclinometer accuracy declining, the error increases for all curve fitting method and the difference among these four curve fitting methods diminishes.

Analysis of the Inclinometer Accuracy. The inclinometer accuracy directly influence the accuracy of measuring data as well as the curve fitting error. Besides, the following hardware design should also depends on the influence of inclinometer accuracy on settlement curve. The inclinometer error is also simulated by normal white noise, and results are shown in Fig. 6.

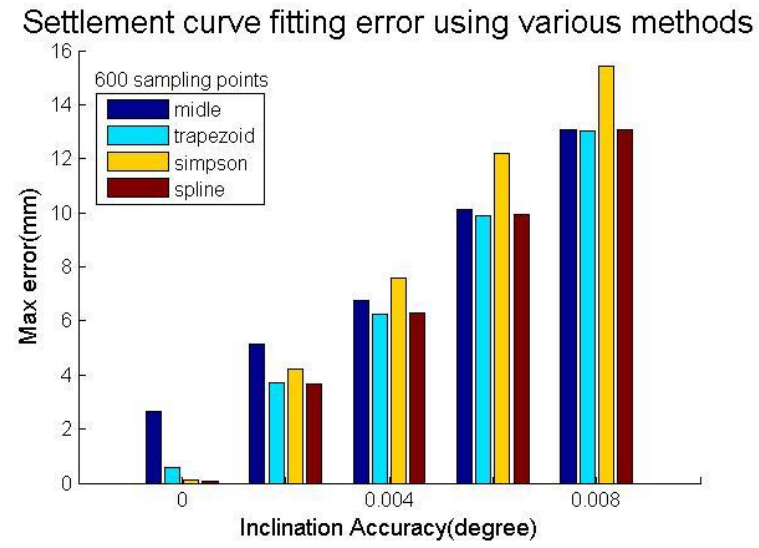

Fig. 5 Histogram of settlement curve fitting error using various methods

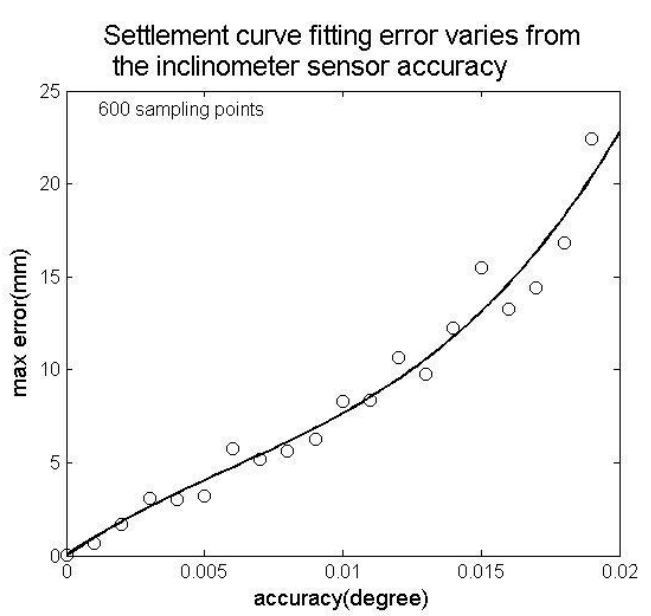

Fig. 6 Settlement curve fitting error varies from the inclinometer sensor accuracy

Fig. 6 indicates that curve fitting error nonlinearly increases with inclinometer accuracy declining. Settlement calculation error is accumulated using inclinometer data, so inclinometer accuracy puts great impact on the final curve fitting error.

Analysis of Sampling Space. Different sampling spaces are used to do longitudinal settlement curve fitting using the curve fitting method based on cubic spline method, which is shown as Fig. 7. 


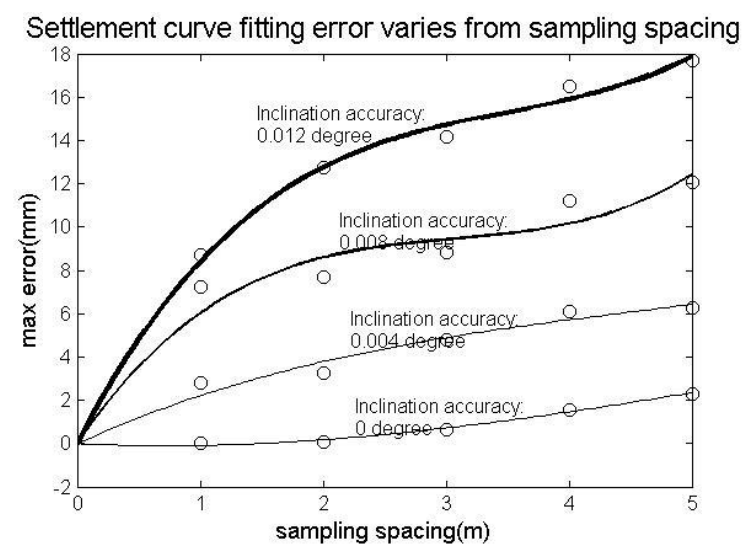

Fig. 7 Settlement curve fitting error varies from sampling spacing

Fig. 7 shows that curve fitting error increases as the sampling space enhances. Diminishing the sampling space can be used to enhance the curve fitting accuracy, which is more effective when using more accurate inclinometer.

Analysis of the Rectification Using Elevation Data at End Points. According to the characteristics of tunnel structure, the elevation at end points of tunnel section is known, so we can use the elevation data at end points to do rectification in order to enhance curve fitting accuracy (Fig. 8 and Fig. 9).

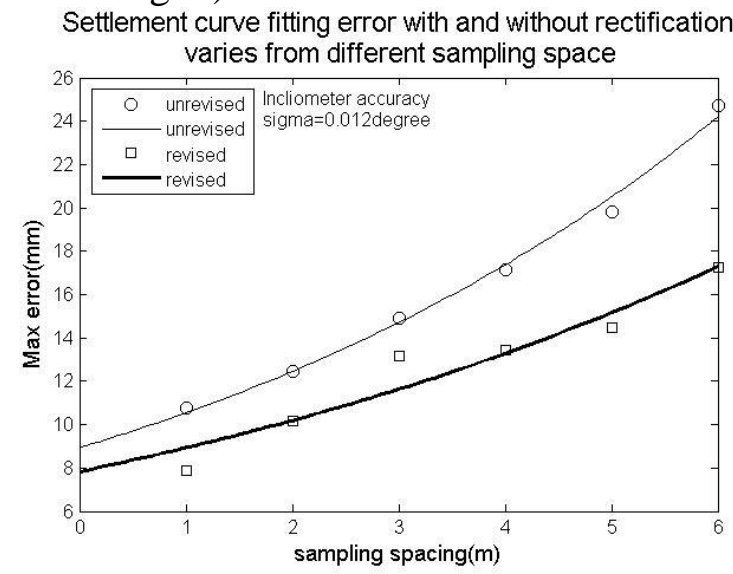

Fig. 8 Settlement curve fitting error with and without rectification varies from different sampling space

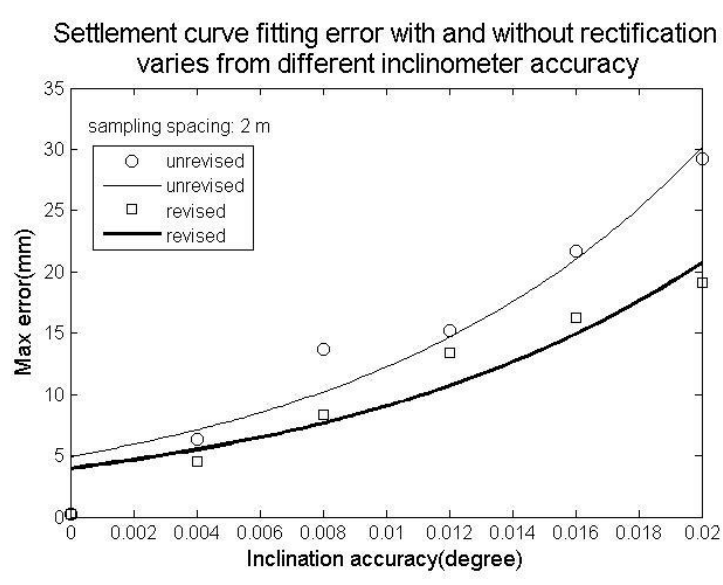

Fig. 9 Settlement curve fitting error with and without rectification varies from different inclinometer accuracy

Fig. 8 and Fig. 9 indicate that the curve fitting accuracy can be enhanced after the rectification using the elevation data at end points of tunnel section. And the rectification is more effective with sampling space increasing and inclinometer accuracy declining.

\section{Technical parameters determination}

At present, second grade levelling, which means monitoring error per kilometer should below 2 $\mathrm{mm}$, is widely used in longitudinal settlement monitoring of shield tunnel. According to the analysis of influence of various factors including sampling space, inclinometer accuracy and curve fitting method, technical parameters are determined after considering the state of technical level (see Table $1)$. 
Table 1 Technical parameters

\begin{tabular}{cc}
\hline Sampling space $(\mathrm{m})$ & 0.2 \\
\hline $\begin{array}{c}\text { Standard Deviation of } \\
\text { error for inclinometer }\end{array}$ & $0.005^{\circ}$ \\
\hline Curve fitting method & $\begin{array}{c}\text { The curve fitting method based on } \\
\text { cubic spline method }\end{array}$ \\
\hline
\end{tabular}

Based on the parameters in chart 1, multiple simulations are conducted and prove that less than $5 \%$ of curve fitting error are more than $2 \mathrm{~mm}$.

\section{Conclusions}

(1) This paper presents a kind of real time monitoring method for longitudinal settlement of shield tunnel using Wireless Sensor Network (WSN) and Micro-Electro-Mechanic System (MEMS).

(2) The error of the curve fitting method based on the cubic spline method is the minimum among the four curve fitting methods.

(3) The influence of many factors, including inclinometer accuracy, sampling space and rectification using elevation data at end points, on curve fitting error are analyzed by simulation. Results show: a) The curve fitting error nonlinearly increases with inclinometer accuracy declining. b) The curve fitting error increases as the sampling space enhances. Diminishing the sampling space can be used to enhance the curve fitting accuracy, which is more effective when using more accurate inclinometer. c) The curve fitting accuracy can be enhanced after the rectification using the elevation data at end points of tunnel section. And the rectification is more effective with sampling space increasing and inclinometer accuracy declining.

(4) Technical parameters are determined based on present monitoring requirement of shield tunnel and the state of technical level.

\section{Acknowledgments}

This work was supported by National Natural Science Foundation Committee program (Grant No.51278381), the National Basic Research Program of China ("973" Project)(Grant No. 2011CB013803) and Shanghai outstanding academic leaders program (Grant No.12XD1405100). Hereby thanks to these programs.

\section{References}

[1] Li, M. and Liu, Y. Underground structure monitoring with wireless sensor networks[A], Proceedings of the Sixth International Symposium on Information Processing in Sensor Networks (IPSN'07), Cambridge, Massachusetts, USA, 2007.

[2] Yoshinori Iwasaki.General Report TS-3A: Instrumentation in Geotechnical Engineering[R]. Proceedings of the 17th International Conference on Soil Mechanics and Geotechnical Engineering, 2009, 1: 3288 3302.

[3] Stajano, F., Hoult, N.A., Wassell, I., et al. Smart bridges, smart tunnels: transforming wireless sensor networks from research prototypes into robust engineering infrastructure $[\mathrm{J}]$. Ad Hoc Networks, 2010, 8(8):872-888.

[4] Kenichi Soga. Micro-measurement and monitoring system for ageing underground infrastructure [J]. Contaminant Hydrology, 2010, 94(3-4), 215-234. 
[5] Zhang Wei, Huang Hongwei. Feasibility study for evaluating convergence deformation of shield tunnel by inclination of segments. ICCES12: Proceedings of the 8th International Conference on Computer Engineering \& Systems, Greece, 2012.

[6] F.Wang, D.M. Zhang, H.H. Zhu, et al. Impact of Overhead Excavation on an Existing Shield Tunnel: Field Monitoring and a Full 3D Finite Element Analysis. CMC: Computers, Materials \& Continua, 2013, 34(1): 63-81.

[7] ZHANG Jiankun, XU Junfeng, XU Guoshuang. Application of hydrostatic monitoring system of the Third Party Monitoring. Modern Urban Transit, 2011(In Chinese)

[8] DAI Jiadong, ZHU Weihong. Application of hydrostatic monitoring system in engineering measurement. Low Temperature Architecture Technology , 2011, 158(8): 104-105 (In Chinese)

[9] WANG Rulu. Application of electronic level meter in metro monitoring engineering. Underground Engineering and Tunnels, 2003 (In Chinese)

[10] XU Xiangqi. Application of electronic level meter settlement automatic telemetry system in metro monitoring engineering. Geotechnical Engineering World, 2009, 12(2): 71-74 (In Chinese) 